\title{
Music Creation by Example
}

\author{
Emma Frid \\ Sound and Music Computing, \\ KTH Royal Institute of \\ Technology \\ Stockholm, Sweden \\ emmafrid@kth.se
}

\author{
Celso Gomes \\ Creative Intelligence Lab, \\ Adobe Research \\ Seattle, USA \\ cegomes@adobe.com
}

\author{
Zeyu Jin \\ Creative Intelligence Lab, \\ Adobe Research \\ San Francisco, USA \\ zeyin@adobe.com
}

\begin{abstract}
Short online videos have become the dominating media on social platforms. However, finding suitable music to accompany videos can be a challenging task to some video creators, due to copyright constraints, limitations in search engines, and required audio-editing expertise. One possible solution to these problems is to use AI music generation. In this paper we present a user interface (UI) paradigm that allows users to input a song to an AI engine and then interactively regenerate and mix AI-generated music. To arrive at this design, we conducted user studies with a total of 104 video creators at several stages of our design and development process. User studies supported the effectiveness of our approach and provided valuable insights about human-AI interaction as well as the design and evaluation of mixedinitiative interfaces in creative practice.
\end{abstract}

\section{Author Keywords}

Music Generation; Artificial Intelligence; Mixed-Initiative Interaction; Algorithmic Composition

\section{CCS Concepts}

-Applied computing $\rightarrow$ Sound and music computing; -Human-centered computing $\rightarrow$ User centered design; Interaction design;

\section{INTRODUCTION}

Short videos such as trailers, vlogs, commercials, event announcements and tutorials provide fast-pacing and engaging experiences to content consumers. However, on the creator's side, obtaining and editing music for such media may be challenging for some, especially for those without audio expertise. Due to copyright constraints, many video creators rely on music that can be downloaded from stock libraries or public domain. A common practice is to use copyrighted music as placeholder and then search for songs that serve the same functionality. This process is time-consuming and users may not find the songs they like. If the source of

Permission to make digital or hard copies of all or part of this work for personal or classroom use is granted without fee provided that copies are not made or distributed for profit or commercial advantage and that copies bear this notice and the full citation on the first page. Copyrights for components of this work owned by others than ACM must be honored. Abstracting with credit is permitted. To copy otherwise, or republish, to post on servers or to redistribute to lists, requires prior specific permission and/or a fee. Request permissions from permissions@acm.org.

CHI '20, April 25-30, 2020, Honolulu, HI, USA.

(C) 2020 Association for Computing Machinery.

ACM ISBN 978-1-4503-6708-0/20/04 \$15.00.

http://dx.doi.org/10.1145/3313831.3376514 music is not correctly documented, posting songs with the wrong type of licenses at e.g. Youtube can also result in considerable fines and removal of the video. Furthermore, existing solutions for music search is primarily based on keyword queries focusing on genre (e.g. "cinematic" and "pop") or emotions (e.g. "sad" and "energetic"). Therefore, video creators may have to scroll through a large selection of songs in order to find an appropriate track. Since keyword tags can be more or less representative for a song, the process of finding suitable music can easily become tedious. In addition, stock music often comes with a fixed duration that may not match the desired video. There are audio libraries that allow the user to download several tracks with different duration and instrumentation, but such music services are usually rather expensive. Consequently, the range of music material available to video creators is somewhat limited, resulting in a homogeneous sonic landscape.

Recent advances in machine learning and artificial intelligence (AI) open up the possibility to generate professional-sounding music for video production. A number of existing solutions requiring various levels of human involvement in the music generation process are available. On one side of the spectrum there are AI music generators that can create music parts of high audio quality (see e.g. [3, 71]). However, such tools often require machine learning and programming skills to operate. There are also procedural music generators (see e.g. [54]) providing interfaces that allow for customization of computergenerated compositions, but such tools require substantial music theory knowledge. While there are examples of interfaces that provide both autonomous AI music generation and a high level of customization and control (see e.g. Orb Composer [30]) such applications may come at the cost of a steeper learning curve. On the other side of the spectrum, there are examples of services developed for non-musicians and those without programming and AI experience (see e.g. Aiva [68] and Boomy [21]). However, these tools mainly allow users to specify a limited set of parameters or keywords for music generation, resulting in the same type of issues as the ones described for music search, see above.

In this paper we explore a new interface solution that bridges AI solutions with non-expert users, balancing between automation and control, thereby making music generation straightforward and easily accessible. We present a new user interface (UI) paradigm for music creation that allows users to provide an example song as input to an AI music system. 
This approach, which we here refer to as "music creation by example", enables video creators to create and customize machine-generated music without going through the process of specifying abstract keywords (such as mood and genre) or learning complicated composition interfaces. Instead, the user provides a piece of music as input to the music generation. This reference can be any song, including copyrighted music. An analyzer extracts musical features from the supplied song(s) and operates an AI music generator to create a set of alternative renditions serving the same functionality as the provided example. The generated tracks have multiple musical features associated with them, such as tempo, baseline, chords and melody. The users can then mix and match these musical aspects, and/or add more reference songs to have wider variety of musical aspects to combine.

The final design of the system described in this paper was verified by extensive user studies, presented in subsequent sections. We identify three contributions to the field of $\mathrm{HCI}$ in the context of this work:

1. A user interface (UI) paradigm for music creation, informed by a user centered design approach

2. A prototype system that has proven to successfully allow users to create songs in collaboration with an AI music system

3. Insights and findings from user studies that can inform the design of mixed-initiative interactions [4] and research on human-AI interaction in creative practice

In the current study we focus mainly on video creators that are musical novices or amateur musicians. However, the proposed UI paradigm could be employed in variety of mixed-initiative interaction contexts.

\section{BACKGROUND}

\section{Human-AI Interaction}

AI techniques can be used to generate new ideas by producing novel combinations of familiar ideas, by exploring the potential of conceptual spaces, and by making transformations that enable the generation of previously impossible ideas [12]. In this study we focus on mixed-initiative interactions between a computer system and human. Mixed-initiative interaction refers to "a flexible interaction strategy in which each agent (human or computer) contributes what it is best suited at the most appropriate time" [4]. This duality can be understood in the following manner: the AI (computer) try to model the way a human thinks, in order to create a system that can perform intelligent actions, whereas HCI research focuses on designing computer interfaces that leverage off a human user in order to help the user in execution of intelligent actions [74].

The increased presence of artificial intelligence in the field of Human Computer Interaction raises many questions about design and evaluation of systems involving humans and AI. A set of guidelines for human-AI interaction was recently published in [7]. In this work, 18 principles roughly categorized by when they are likely to be applied during interaction with users (initially, during the interaction, when something is wrong and over time) were presented. Guidelines included: making clear what the system can do and how well it can do it; timing services based on context; showing contextually relevant information; matching relevant social norms; mitigating social biases; supporting efficient invocation, dismissal and correction; scoping services when in doubt; making clear why the system did what it did; remembering recent interactions; learning from user behavior; updating and adapting cautiously; encouraging granular feedback; conveying consequences of user actions; providing global controls and notifying users about changes. In this study, we employed the above described guidelines for evaluation of our prototype.

\section{Computational Creativity}

New technologies are drastically changing the nature of creative processes. Today, computers are increasingly becoming essential tools in creative practice. The term "computational creativity" refers to the study of building software that exhibits behavior that can be deemed creative in humans [18]. Several books on the topic of computers and creativity have been published, see e.g. [13, 48]. Computational creativity research has explored autonomous generative systems in a wide range of areas, such as math, poetry, painting and music composition [18]. The term "generative" in this context refers to generative design techniques that use algorithmic encoding of domain knowledge to automate parts of a design process [74]. Generative design involves considering the computer as a collaborative partner in the design process capable of generating ideas and stimulating solutions in response to models of design conditions and performance [64]. Due to the nature of generative design systems, it has been suggested that generative prototyping systems should act as brainstorming tools for producing creative prompts, i.e. rough designs, which can later be refined using human design workflows [74].

In recent years, machine learning algorithms have been adopted as creative musical tools, motivating a new line of algorithms known as interactive machine learning [27]. It has been hypothesized that machine learning will blur the line between music consumption and music creation [5]. However, several concerns related to automation of creativity have also been raised. For example, some express scepticism about the creative potential of computer software, since they believe it to be impossible to simulate creative thinking using algorithms or information processing systems [67]. Others have described creativity as something "mysterious" which is "seemingly impossible to teach" [61]. On the other hand, counter-arguments based on the idea that humans are, on some level, only capable of doing what we were designed or programmed by evolution to do, have also been presented [61]. Interestingly, some claim that creativity is not beyond scientific study and that research on computational creativity may contribute to the understanding of human creativity, allowing us to produce programs where the software acts as a creative collaborator rather than a mere tool [18].

\section{Al Music}

Algorithmic composition, i.e. creating music through the use of algorithms, is a field motivated by "extend[ing] musical thinking through formalised abstractions" [49]. It involves partial or total automation of music composition processes through the use of computers [26]. The area has been studied 
extensively for over fifty years [8] and widely discussed within the New Interfaces for Musical Expression (NIME) [57] and Computer Music communities (see e.g. [56, 51, 60] for an overview). Interestingly, algorithmic music has been described as a composition tool modeling creativity as an algorithm [33]. Notable contributions include David Cope's "Experiments in Musical Intelligence" (EMI) project [19], which focused on emulation of styles of specific composers, and Iannis Xenakis' [75] piece "Analogique", which made use of Markov chains. Overviews of the history and principles of algorithmic composition are presented in $[47,20,50]$. While work on modeling music using neural networks was published as early as in 1988 [11], more recent advancements in the field of deep learning have paved the way for a number of new methods for music generation (see [14] for an overview).

Current deep learning systems for music generation focus largely on autonomous generation; little work in this domain has yet addressed issues related to human control and interaction [14]. The importance of user centered design processes has been stressed in the context of interactive machine learning [10]. When it comes to evaluation of music generation systems, focus has traditionally been on comparing musical outcomes using Turing tests rather than on improving human$\mathrm{AI}$ interaction; if the generated sounds sound like (or are preferred to) human output, the machine has succeeded [8].

Algorithmic music raises question about the musicality of system-generated music and the aesthetics of AI-generated material in general (see e.g. [46]). Even if today's generative models are quite sophisticated, they usually do not include an element of reflection or evaluation; it is not uncommon that these systems rely on human designers to preside over matters of aesthetics [2]. This discussion relates to the concept of functional music, so called Muzak [35], which has often been criticized to have no aesthetic value and also result in composers losing commissions. Similar claims are sometimes made for AI music. A discussion on algorithmic music for mass consumption and universal production was presented in [38]. Interestingly, in a recent study on AI versus stock music conducted on 626 video creators, users had no preference between AI versus human-generated music for video editing [53]. Some attempts towards developing metrics for evolving musically satisfying results from computergenerated music compositions have been made (see e.g. [15]). The need for a more systematic evaluation of musical metacreation has been stressed for example in [2]. Further investigations in this field are required. We want to emphasize that the aim of the current work is not to replace the role of the composer, rather, it is to make AI music more accessible to non-musicians through $\mathrm{HCI}$.

\section{Related Work - Al Music for Video}

When it comes to sound and music for film, professional video productions usually rely on composers to create background music and Foley artists [6] to create sound effects. For content published on social platforms, numerous stock music libraries provide download services for background music. Examples include Marmoset [43], Epidemic Sound [66], Musicbed [55], Audio Jungle [45], Premium Beat [65], Bensound [69],
Soundstripe [42] and SmartSound [37]. Up to this point, little work has focused on automatically generating music based on video content, but there are examples of research focusing on soundtrack recommendation based on mapping low-level acoustic and visual features into an emotional space [72, 63, $39,40]$ and automatic editing of user-generated videos into music-compliant professional-like videos, using soundtrack recommendation [41].

When it comes to computer-based music generation based on deep learning, two main types of systems can be distinguished: 1) autonomous music-making systems aimed at creation of original music for commercials and documentaries (e.g. Amper Music [52] and Jukedeck [36]), and 2) computerbased environments based on assisting human musicians (e.g. FlowComposer [59] and OpenMusic [9]) [14]. To our knowledge, only one software solution using AI to generate music for video exists: Muzeek [32]. There are however systems that enable music creation for video without using AI methods, such as Filmstro [28]. Moreover, certain software solutions provide features for customizing song duration, something that is very useful for video purposes (see e.g. SonicFire [44] and Audition [1]). However, as of today, none of the above-mentioned systems allow a user to select a reference song to guide the process of music generation, which is the focus of the work presented in this paper.

Although the idea of design by example has been employed in other research domains, e.g. in the use of templates in design and fabrication [62], it has not yet been widely adopted in the music domain. Previous work employing such a method in a music context include work presented in [23], generating highquality expressive solo performances of jazz ballads based on examples of human performance, and the MorpheuS system, generating new musical pieces based on rhythms, repeated patterns, and tension profiles from an example piece [29].

\section{PRESTUDIES}

\section{Questionnaire}

\section{Method}

We initially conducted a formative study to better understand users' needs in the workflow of adding music to videos. The study was designed as an anonymous online questionnaire distributed to video creators (making Youtube videos or vlogs) through the User Testing platform [70], an online crowdsourced user study system. A total of 30 participants (17 F, $13 \mathrm{M}$ ) completed the survey. Average age was 33.9 years (median 26.5, $\min 19.0$, max 72.0 years). The majority of the participants $(n=26)$ were from the US, 2 from Canada and 2 from the UK. User screening was done based on content creation type (vlogs and Youtube videos) and if users added music to their videos using stock libraries or other services. Users composing or producing music for their own videos were not allowed to participate.

The questionnaire consisted of multiple-choice questions focusing on the use of audio in the context of video creation and open-ended questions on the same topic. Multiple-choice questions were analyzed using frequency metrics (counts/total number of participants). Open-ended questions were analyzed 
using thematic content analysis [73] with category counts; free text answers were initially encoded into different categories based on keywords. Answers related to similar keywords were grouped together and occurrence for respective keyword category was calculated. The most frequent categories are presented in the Results section below. The questionnaire and data are available as supplementary material.

\section{Results}

Participants described that they used a wide range of different software solutions for video and audio editing. The most commonly used softwares were iMovie (10/30) and Adobe Premiere Pro (8/30). In total, 22 participants described that they used music for all aspects of their videos (background music, intro/outro and clips in the middle) while 8 participants used it only for background music. On average, the participants stated that they needed 4.67 new songs for their videos per month. They used a wide variety of different music genres in their videos, but the majority stated that they rarely or never used sound effects (19/30). Most participants used free music libraries (24/30), while only a few used paid premium (4/30) or paid royalty-free (2/30) music libraries. The most commonly used music library was YouTube Audio Library [76] (21/30), but a total of 20 different services were mentioned. Participants stated that they either did not use algorithmic music (12/30), were not sure about whether their music was algorithmic or not (10/30), or did not know what the term algorithmic music means (8/30).

We identified three themes based on descriptions of user's workflow when adding music to videos. Participants mentioned editing the duration of a video or trimming and syncing music to their video (12/30), adding music as a final step in their video editing (11/30), and adjusting audio level of the music (5/30). Interestingly, three major challenges encountered when searching for and mixing music for videos were discussed: searching for a musical piece/finding appropriate music (mainly in terms of duration, but also mood) (15/30), license/copyright/having to purchase music (9/30) and timeconsuming editing processes (change tempo/BPM, cut and trim, pitch shift, change duration, sync to video, adjust volume) (7/30). When asked what would make the music editing process easier, the following suggestions were mentioned: 1) a large library of music (9/30); better search/filtering/labeling of music (8/30); an all-in-one-tool, i.e. a library of sounds within the editing tool, including search and download features (5/30); royalty free music without copyright issues (5/30); making trimming/duration adjustments easier (4/30); free music (4/30) and better user interfaces $(3 / 30)$.

\section{Brainstorming Workshop Method}

Knowing the need for music in the context of video creation, we conducted an informal workshop with HCI researchers to seek guidance for the interface design of the envisioned system for music generation. A total of 21 researchers ( 8 female, 13 male) participated. They were all working in the same lab at a large tech company (this was the only part of the study that involved researchers instead of actual video creators, although one of the researchers was in fact also a video creator).
The workshop started off with a presentation of the project, including previous and related research, intended user group (novices with limited musical background) and demonstrations of design suggestions (mockups) of the interface. Examples of proposed interface solutions included canvas GUIs allowing you to draw music using strokes or selections in a grid to generate music on a video timeline. The following questions were presented as discussion points: 1) possible interaction strategies for music creation, 2) methods for defining desired functions of a music creation tool, 3) musically relevant features extracted from video and 4) using iterative and user-centered design methods in the context of the current project. The session lasted for 1 hour (15 minutes of this was dedicated to a project introduction). Feedback and discussions were transcribed by the first author, who then summarized the discussion taking place, extracting quotes that could be relevant for further development of the prototype.

\section{Results}

A number of interesting ideas were brought up during the brainstorming session. One conclusion that could be drawn was that we needed a more in-depth understanding of the user's workflow. A second study with video content creators was thus conducted, as described in the next section. Another interesting discussion point was that it might be fruitful to focus mainly on modification of high-level aspects of the music, rather than on low-level ones, since the intended user group consists of musical novices. For example, users might know which emotion that the music should convey, but not be interested in tuning frequencies or defining rhythmic structures. Enabling "orchestration of different layers of the music" was mentioned in the same context.

One suggestion that was brought up was to develop a system that would allow for mixing of certain aspects of the music, thereby giving users creative control and freedom, without requiring prior music knowledge. Interestingly, the participant who was also a video creator described: "A lot of times when I am editing videos for myself or for other people, when it comes to the score, usually someone will be like 'I want something that sounds like this', and then they give me an example of another video. So, I feel like that being able to say (...) I want music like this, and then from there you could get this generation (...) these building blocks that are generated based on a type of song or music that you chose." This participant also mentioned that some people she worked with would hum a melody to describe the music that they wanted for their video. Another participant said: "I can imagine that you input your inspiration through examples [links, sounds, images]... what are the parts that I like, and can I generate something based on that?" Based on this idea, participants discussed having a mood board or a "Pinterest of Songs" as starting point for music generation.

\section{Interaction Scenarios \\ Method}

In order to explore possible design directions, an additional user study with video content creators was conducted using an online questionnaire. It has been shown that parallel prototyping, i.e. generating multiple prototypes that can receive 


\begin{tabular}{lll}
\hline & Advantages & Disadvantages \\
\hline A) & make music quickly (14) & lack of customization (18) \\
& no copyright or fees (11) & output too similar to input (5) \\
simple/straight-forward (12) & sound not original/unique (5) \\
B) & $\begin{array}{l}\text { customization/creative control (23) } \\
\text { creating remixes/hybrid songs (18) }\end{array}$ & poor selection $\rightarrow$ bad results (11) \\
& too complex/time consuming (8) \\
C) & good if a song is stuck in your head (16) & requires music skills (7) \\
& requires music skills (14) \\
& easy/quick (8) & doubt that it would work (11) \\
& you can specify tempo/tone (7) & requires melody idea (9)
\end{tabular}

Table 1. Advantages and disadvantages of respective scenario, where A) music generation based on one example song, B) music generation based on a selection of example songs and mixing layers/properties of this music, and C) music generation based on humming or whistling a melody. Numbers in brackets correspond to number of participants that mentioned respective theme.

feedback in parallel, leads to better design outcomes [24]. Based on ideas from the brainstorming session, we defined three possible design scenarios for music generation and evaluated them with video creators. Each scenario had a different input method for music generation: A) music generation based on one example song, B) music generation based on a selection of example songs, allowing the user to mix layers/properties of the generated music, and C) music generation based on humming or whistling a melody. The Interaction Scenario questionnaire was distributed on User Testing, using similar screening methods as for the previous questionnaire. A total of 60 video creators $(37 \mathrm{~F}, 23 \mathrm{M})$ participated in the study. Average age was 30.9 years (median 30.0, min 18.0, max 63.0 years). In terms of musical experience, 26 did not play any musical instrument or sing, 25 were amateur musicians and 9 were musicians with several years of practice. Most of the participants were from the US (47/60), followed by the UK $(9 / 60)$, Canada (3/60) and Belgium (1/60).

The participants were initially asked to discuss which musical aspects that they considered when searching for songs for their videos. We hypothesized that this information could be relevant for the interface design as well as the selection of control parameters for music generation. Then, the different interaction scenarios were presented in the form of short video clips, together with descriptive prompts and music examples for respective workflow. The videos, as well as the online questionnaire presented together with them, are available as supplementary material. In order to reduce the risk of carryover effects, presentation order was randomized so that all conditions (i.e. order of scenarios) occurred for 10 participants each. For each scenario, participants described potential advantages and disadvantages of respective model. In the end, participants were asked to select which scenario they preferred. For interaction scenario A and B, we also asked participants if they would prefer providing example music in the form of generic (i.e. unknown) or well-known songs. Analysis of free text data was done using similar methods as for the previous questionnaire.

\section{Results}

Participants stated that they mainly considered emotion/mood (56.7\%), genre (18.3\%) and tempo (13.3\%) when searching for music. Interestingly, aspects such as instruments and composition (e.g. temporal structure such as intro, bridge, chorus and outro) as well as chord progression were less commonly mentioned. A summary of advantages and disadvantages for respective interaction scenario is presented in Table 1. Interestingly, the most commonly preferred interaction strategy was B), the method in which music was generated based on a selection of songs, combined with a grid interface allowing the user to mix and match different properties (tempo, instrument and mood) of generated AI music ( $\mathrm{n}=31,51.7 \%)$. The second most preferred option was A), i.e. to provide one example song $(\mathrm{n}=17,28.3 \%)$. The vast majority of the participants preferred providing a well-known song as example reference, instead of using a generic song. The least preferred option was C) to whistle or hum an input melody $(\mathrm{n}=12,20.0 \%)$.

Overall, scenario B) was preferred because of its high level of creative control and customization capabilities, the possibility to select several input references and create remixes or hybrid songs, as well as allowing for creation of unique, new and personal sounds. One participant stated the following about this interaction scenario: "The workflow itself is easy to understand, and almost fun in a way, giving you more control of a largely automated process. It makes you feel as you are helping in the creation process instead of solely relying on the machine." In general, there were many positive comments about the interactive grid presented in this interaction scenario, which allowed users to select mood, tempo or instrument for respective track. One subject stated: "Getting to decide which parts of which generated tracks and then throwing it all together is neat. I have never seen anything like that before, where it generates [music] itself, as opposed to having to create it yourself."

\section{SUMMARY OF FINDINGS}

The three studies described in previous sections led to the following conclusions:

- Users' major challenges relate to finding appropriate music, copyright and editing (mainly duration and volume)

- Users would benefit from having access to a larger library of music, better search tools and better labeling of music, and an all-in-one music and video editing tool

- Users do not use algorithmic music or do not know what it is

- Novice users might want to focus mainly on higher level properties of the music

- Users might benefit from giving examples, or mood boards, of songs that remind of the sound that they are striving for

- Users want to create music in collaboration with an AI, thereby remaining a certain level of creative freedom, customization and control

- Users would prefer having the ability to provide the music system with several examples of well-known songs

- A grid interface allowing for mixing and matching of different aspects of AI-generated tracks might be one potential solution for customization of AI music

\section{SYSTEM}

\section{Design Process}

We used a user centered design approach; decisions were informed by user studies and relied on user-centered design methods and iterative prototyping. An overview of the 

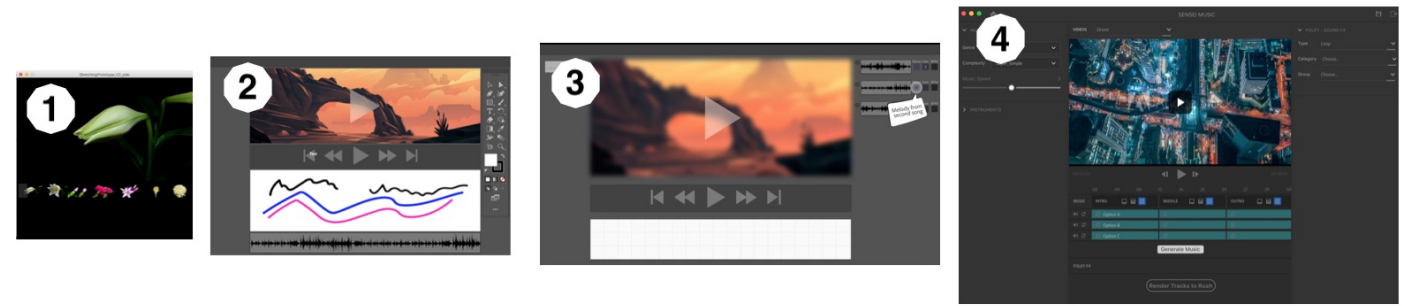

Figure 1. Overview of the interface design for different iterations of the prototype. Interface 1 and 2 made use of a drawing metaphor for music generation. This interaction paradigm was rejected since none of the initial studies appeared to support such an approach. The preferred scenario from the Interaction Scenario study was interface number 3. Apart from allowing the user to input a song to the music generation, this GUI also included a grid interface for customization. The first version of the developed application is presented in interface 4.

different versions of the prototype system is visualized in Figure 1. In total, 104 video creators took part in this design process; questionnaire participants $(n=30)$, interaction scenario participants $(n=60)$, evaluators $(n=12)$ and expert evaluators $(\mathrm{n}=2)$ recruited from a large tech company. None of the video creators were members of the authors' research lab or had any relation to the researchers. Each step of the development process was informed by user studies. Our first study, the questionnaire, focused on needs of content creators when adding music to videos. Results suggested that it was difficult for video creators to find and edit music based on keywords. The most important aspect to consider in this context was emotion/mood. The users also requested having a service that would provide easy access to free music. Knowing the difficulties associated with keywords as starting point for music search or music generation, we developed a music creation system that would implicitly allow you to embed properties such as emotion into the AI music generation by providing an example song. Based on these findings, as well as suggestions from the brainstorming sessions, we presented three prototype systems (interaction scenarios) to another set of video creators in order to investigate the potential of different input methods for music generation. Results from the interaction scenario study revealed that participants would prefer to input well-known songs instead of singing or humming a melody as input to an AI system. Moreover, the users wanted an interface that allowed for a high level of control and customization. Finally, evaluation sessions resulted in a range of changes, described in detail in the Evaluation section below. The final system design is presented in Figure 2.

\section{Workflow}

The workflow of the final system design is visualized and described in Figures 2 and 3. The user initially selects one or several reference songs that fits the video. Analysis of the selected reference music results in a set of parameters extracted for customization of the music generation. The current system builds on an existing open source AI music solution called IBM Watson Beat [31], a symbolic music generation system based on MIDI. It uses a combination of reinforcement learning and neural networks to create several layers of music in MIDI format based in the following input: a 10-20 seconds long melody file and a set of parameters for a creative engine (e.g. complexity, time signature, tempo in beats per minute, energy and duration, see GitHub repository for details [16]). The output is a set of tracks, or musical elements, which can be mixed and matched in order to create a song for a video through selections in a grid. The grid has different buttons for selection of melody, pads, bass and drums for respective computer-generated track, and allows the user to pick and select various elements and mix them into a final song, which can then be rendered to the timeline. This system enables users to creatively generate unique music that they can use in their videos without the risks of copyright infringement, as the music generation is only inspired by example songs, not regenerating copyrighted material.

\section{System Architecture}

The system consists of an Electron [25] app with built-in IBM Watson functionality. Electron allows for the development of desktop GUI applications using web technologies by combining the Chromium rendering engine and Node.js runtime. The user interface builds on React components. The following parameters are set depending on properties of the input song: genre, tempo and density. The parameters of the music generation of IBM Watson Beat is specified in Python scripts, which in turn output a number of MIDI files. Real-time communication between the Electron app and sound synthesis software is enabled through the use of integrated web sockets in Max/MSP [22] and real-time synthesis of audio is done using a selection of VSTs. Each generated music track was 1520 seconds long. Since the quality of drum patterns generated using IBM Watson Beat was not sufficient, drum patterns were obtained from predefined drum loop sample libraries.

\section{EVALUATION}

Prior to the evaluation of our first prototype, a pilot study was conducted with two researchers from our lab (both male, age 22 and 25, respectively). Observed issues and bugs were then addressed before the actual evaluation study was conducted.

\section{Evaluation of First Prototype Method}

A call for participation for a user study focusing on computergenerated music for video was distributed on the mailing list of a large tech company. In total, 12 employees $(5 \mathrm{~F}, 6 \mathrm{M}$, 1 They), participated. Average age was 31.9 years (median 30.5, min 23.0, $\max 45.0$ years). Out of these participants, 2 were musicians with several years of musical training, 5 were amateur musicians and 5 did not play any musical instrument/sing. In terms of video production and editing 


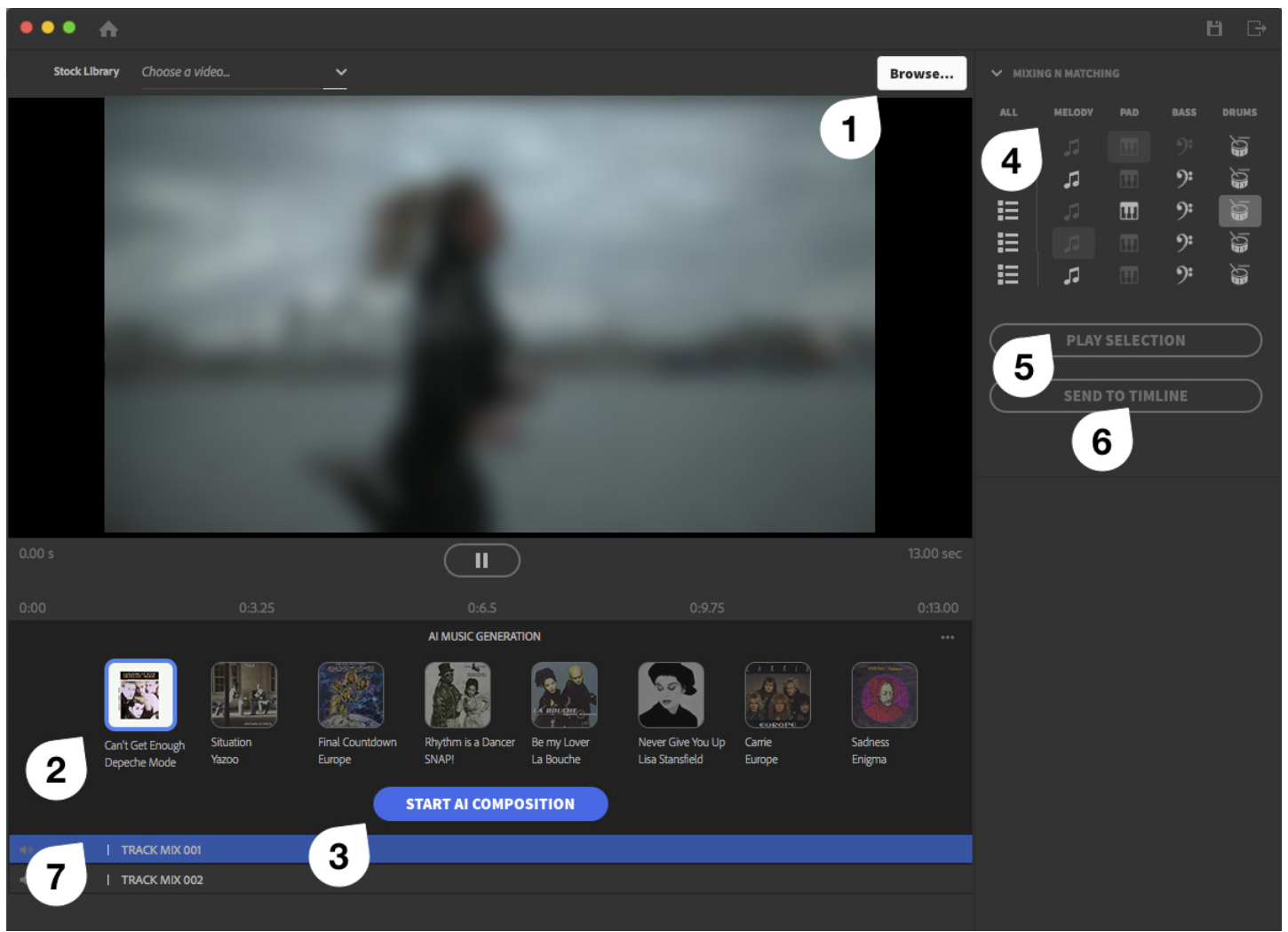

Figure 2. Overview of the workflow and GUI of the final system. 1) Select a video. 2) Select a reference song that goes well with the video. 3) Press to generate several tracks of AI music based on, but not exactly similar to, the reference song. 4) Mix and match melody, pads (sustained chords), bass and drums from different AI-generated tracks. 5) Play a preview of the selected combinations of melody, pads, bass and drums from different AI-generated tracks. 6) Render the mix to the timeline. 7) Your mix shows up here.

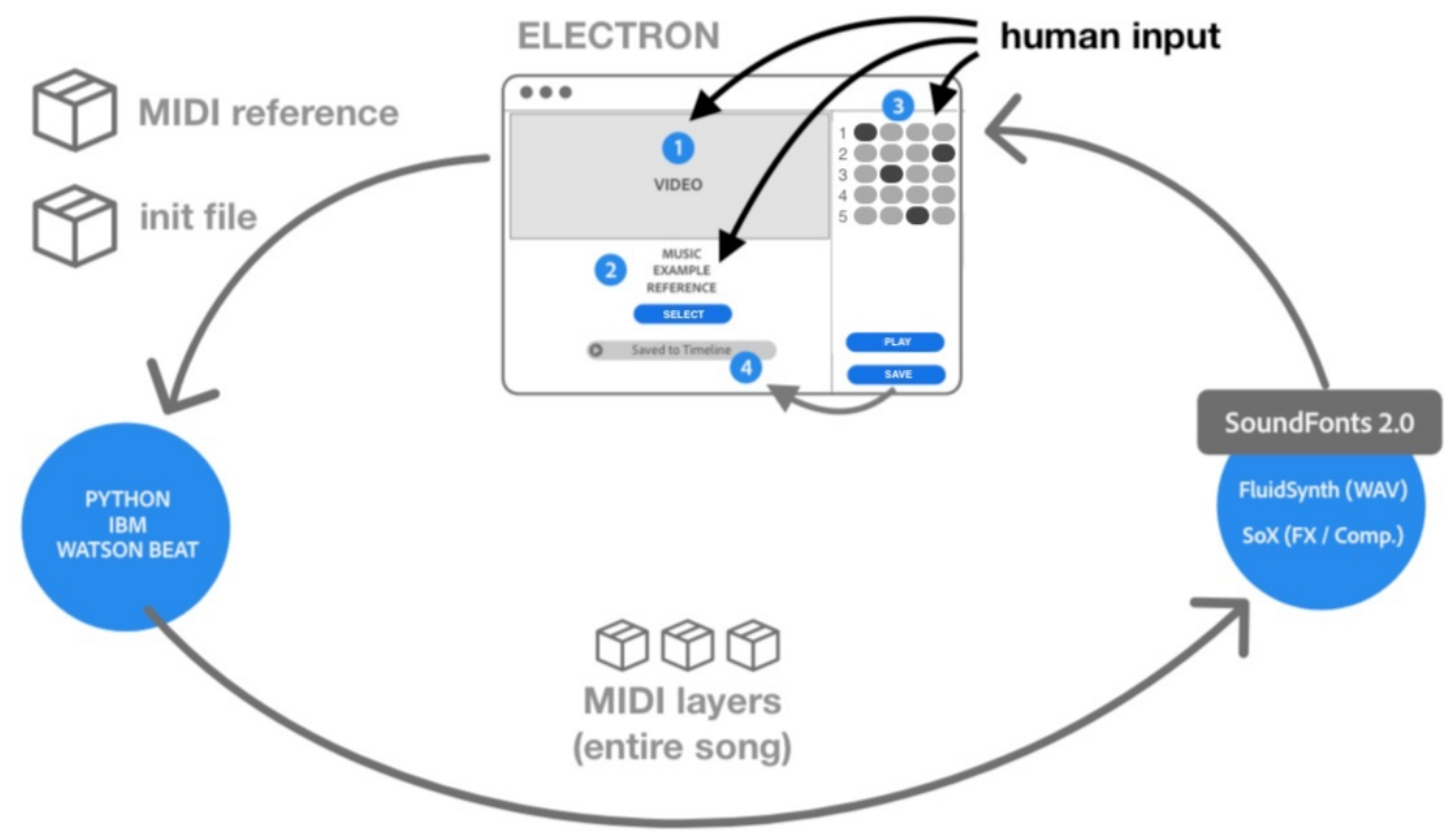

Figure 3. Schematic representation of the pipeline for computer-generated music, with annotations of processes involving human input. 1) User inputs a video. 2) User specifies a reference song. 3) User mixes and matches melody, pads, bass and drums for AI-generated tracks. 4) User saves mix to the timeline. 
expertise, 1 person was an expert who primarily worked with video editing, 4 had professional experience, 4 had some experience and 3 had little experience.

The evaluation sessions lasted for 20-30 minutes per person. The main author acted as test leader for the evaluation sessions. After signing a consent form, the sessions started off with a description of the experimental procedure (instructions were read from a prepared manuscript) and a short demo of the functionalities of the prototype. The sessions, including the screen interactions, were video recorded. Participants were asked to complete the following two tasks: 1) create music for a video based on ONE example song, and 2) create music for a video based on TWO example songs. For two songs, the grid display included more options, but apart from that the procedure was the same. Participants were encouraged to think aloud [17] when solving these tasks.

A semi-structured interview was held after the two tasks had successfully been completed. The following topics were used as starting point: reflections on using existing songs as input for music generation, reflections on mixing and combining different properties of tracks generated by the system (using the grid allowing for customization), level of creative control provided by the system, intuitiveness of proposed workflow and suggestions for additional features that could be implemented in order to improve the system, i.e. what would be required from the software in order for the person to actually use it. Participants received a 10\$ Amazon gift card for their participation.

After the session participants filled out a questionnaire focusing on topics related to the interview questions as well as metrics frequently used for evaluating interactive systems [34] (difficulty of the task, time required to learn the system, level of creative control provided by the system, and how enjoyable it was to interact with the system). Participants were asked to rate these properties on a set of scales labeled from 0 to 10 (with marks for every step), with labels "not at all [adjective]" to "very [adjective]" on opposite ends. This questionnaire, and music generated during these experiments, as well as quantitative data from the evaluation sessions, are available as supplementary material.

After the experiments, think-aloud and interview sessions were transcribed and encoded using the same method as for the initial questionnaire and the interaction scenario study. A summary of qualitative and quantitative findings is presented below.

\section{Results}

A plot of users' ratings of the difficulty of the task is displayed in Figure 4. All participants rated the task as being in the lower end of the difficulty scale. Similar results were observed for time required to learn how to use the system, see Figure 5. Based on the interview results, we concluded that the user experience could be improved for the grid interaction. However, all participants rated the system as being somewhat enjoyable to use, see Figure 6. A plot showing participants' satisfaction with the generated music is visualized in Figure 7. For the level of creative control that the system provided, see Figure 8 . The ratings of this metric varied substantially between participants. Interestingly, analysis of influence of music expertise on ratings of creative control revealed no strong association between the two variables.

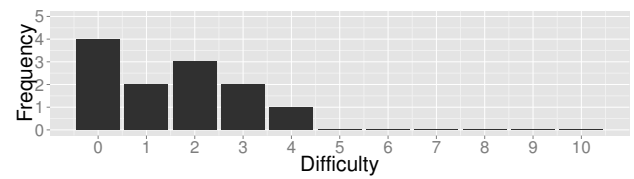

Figure 4. Perceived difficulty of the task, ranging from $0=$ not difficult at all, to $10=$ very difficult.

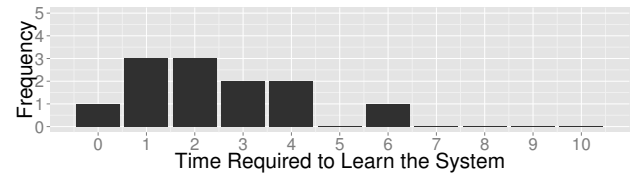

Figure 5. Time required to learn how to use the system, ranging from 0 $=$ no time at all, to $10=$ very long time.

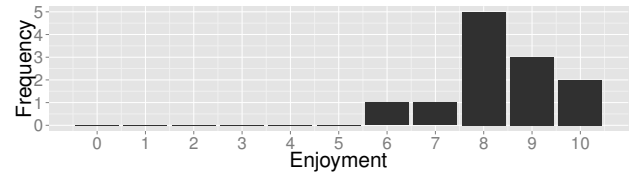

Figure 6. How enjoyable it was to generate music using the software, ranging from $0=$ not enjoyable at all, to $10=$ very enjoyable.

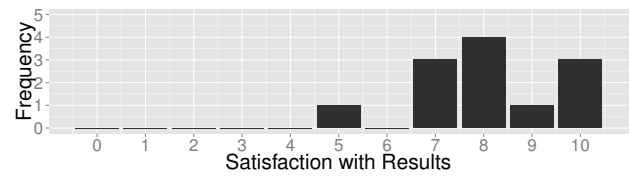

Figure 7. Satisfaction with results, i.e. the generated music, ranging from $0=$ not satisfied at all, to $10=$ very satisfied.

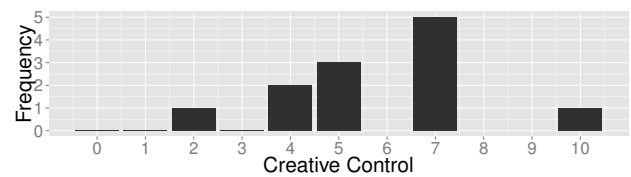

Figure 8. Level of creative control provided by the system, ranging from $\mathbf{0}=$ no creative control at all, to $10=$ very much creative control.

In general, participants appeared to enjoy the interaction with the system. For example, one participant stated: "I feel creative. Like I know [the generated music] is unique." This unique property was said to be the major advantage of the system. Participants also enjoyed the possibility to explore sounds using the grid interface. Another participant said: "I could do this all day; it is so fun! (...) That is so cool. [The AI is] doing all of the hard work in the background for us". Overall, participants were positive about generating music by example. Interestingly, one participant pointed out that “...even something that just helps me finding something similar, without me having any control over messing with it...I mean, that alone is a big deal." Moreover, another subject described that "If [the system] is able to take out different drum patterns and [...] remix the melody, that is something that I am already 


\begin{tabular}{lll}
\hline Feature & Count & $\mathrm{U} / \mathrm{E}$ \\
\hline adjust tempo/BPM & 9 & $7 \mathrm{U}, 2 \mathrm{E}$ \\
volume control for tracks & 5 & $4 \mathrm{U}, 1 \mathrm{E}$ \\
more instrument options & 3 & $1 \mathrm{U}, 2 \mathrm{E}$ \\
multitrack output & 3 & $2 \mathrm{U}, 1 \mathrm{E}$ \\
key/pitch options & 2 & $1 \mathrm{U}, 1 \mathrm{E}$ \\
matching video features to sound & 2 & $1 \mathrm{U}, 1 \mathrm{E}$ \\
loop function & 2 & $1 \mathrm{U}, 1 \mathrm{E}$ \\
define key points/timestamps & 2 & $1 \mathrm{U}, 1 \mathrm{E}$ \\
mood options & 1 & $1 \mathrm{E}$ \\
style options & 1 & $1 \mathrm{E}$ \\
define segments (intro/outro) & 1 & $1 \mathrm{E}$ \\
customize tone & 1 & $1 \mathrm{E}$ \\
waveform visualization & 1 & $1 \mathrm{E}$ \\
adjust duration & 1 & $1 \mathrm{U}$ \\
\hline
\end{tabular}

Table 2. Summary of features suggested by users. $U=$ user test participants (total $n=12$ ) and $E=$ expert evaluators (total $n=2)$.

trying to do on my own [...] in a way that is not as quick and not as clean, where I am just kind of chopping things up, so this is kind of an easier way to do that". She also mentioned that the design by example feature would make her more compelled to use the system. Yet another participant described that he liked the approach a lot, since it made him feel like he was choosing "the mood of the music". A summary of functions and additional features proposed for the system is presented in Table 2.

\section{Changes Based on User Feedback}

Feedback from the evaluation was taken into account when developing the final prototype. In particular, we focused on improving the GUI of the grid, including playback and mixing functions, as well as providing users with a wider range of control. Instead of requiring participants to select one melody, bass and drum tracks for their mix, any combination of tracks was now allowed, including selecting only a drum or a melody. We also added a function to generate sustained chords (pads) for the music (previous options were melody, bass and drums). In addition, the tracks could be regenerated using different instruments. In terms of the GUI, we removed the left input panel and instead focused on the timeline and grid interface for customization. Finally, the new system allowed users to create several mixes and to render them to the timeline, before deciding which one to use for their video.

The final grid interface is displayed in in Figure 9. This $5 \times 4$ grid is created from one song selection (one example). Five different AI music tracks are generated based on one input song. Each row corresponds to one AI-generated track. Different components from respective track can be selected and previewed alone or together. The interface allows users to jump between different combinations of AI music and adjust their selection. If another song is selected for AI music generation, $5 \times 4$ more buttons will be added to the interface. Elements from multiple tracks can thus be combined. The number of tracks and music elements available were informed by user studies as well as research on visual shortterm memory.

\section{Expert Evaluation of Final Prototype}

\section{Method}

In order to gain insights about the possibilities versus limitations of our system, we held informal qualitative evaluation

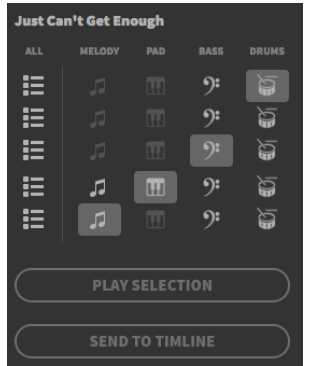

Figure 9. The final design of the grid. Five tracks are here generated based on the song “Just Can't Get Enough". Rows corresponds to AI-generated tracks. Columns correspond to different elements of respective track, such as melody, pads, bass and drums. Users can select any combination of elements and preview them in a mix by clicking "play selection".

sessions with two experts in video production ( $1 \mathrm{~F}, 1 \mathrm{M}$, average age 29.0 years; median 29.0, min 28.0, max 30.0 years). Both participants were recruited from a large tech company. The experts also had some musical experience: one was a professional musician/composer and the other was a musician with several years of musical training. The second author served as test leader and the first author was connected through video-link, observing the sessions, taking notes. We asked the participants to create a set of tracks for one video of their choice. As we had already confirmed that the system fulfilled general usability requirements (e.g. difficulty of the task etc.) as well as quality of the musical outcome, this final evaluation focused more on the human-AI interaction. For this purpose, we used [7] as a basis for evaluation.

The evaluation sessions began with a short walk-through of the system's workflow. The evaluators were asked to compare our system in relation to a baseline case, i.e. another system enabling creation of AI music. We used the Aiva [68] music generation service for this comparison. The Aiva system allows users to create music by defining the following parameters in a web interface: 1) preset style (e.g. modern cinematic, sea shanty, jazz etc.) 2) key signature, time signature, pacing, instrumentation, duration and number of compositions. The system then generates the specified number of tracks according to selected parameters. One participant started off using our system, and one participant started off using the Aiva system. For both systems, the task was to create a song. The evaluation based on human-AI guidelines was only performed for our system. For this task, participants were provided with a table from [7] with examples of respective guideline. A semi-structured interview focusing on advantages and disadvantages of respective system was conducted at the end of the sessions. The evaluations lasted for approximately one hour and participants got two 10\$ Amazon gift cards for their participation.

\section{Results}

The 18 human-AI guidelines were useful for our evaluation purposes. The guidelines served as a tool for identifying issues related to the music generation process, as well as the level of feedback about AI processes communicated to the user. Aspects that were identified as potential issues by both participants were: support for efficient dismissal, scope 
services when in doubt and make clear why the system did what it did. In general, both participants mentioned that they would want to provide more (creative) input to the system. A summary of proposed features for a future system is presented in Table 2. The first participant mentioned that he did not think that the system provided very much control, but he said that this was not an issue since there were so many options to choose from. In general, this user was very positive about the possibilities provided by the system. He suggested more customization capabilities for the timeline, and did not necessarily want the AI to take over those parts. Similarly, the second participant emphasized the importance of being able to define "key points" or timestamps where the music should change, and to allow the AI system to help with aspects related to timing and synchronization. Moreover, both participants mentioned that it would be nice to be able to adjust levels of respective music component, in order to decide which sounds that should be more prominent in the mix.

When comparing our prototype system with Aiva, the first participant mentioned that the baseline interface made him feel "less in control" and that it felt like the entire timeline was already defined, describing the music as "an entire thing that I have to dig into". Interestingly, the second participant was more positive to Aiva, focusing mainly on the advantages of having a set of presets for instruments (e.g. specific band constellations). However, she mentioned that it would be interesting to have a more "human focused" way of referring to sounds. For example, it would be better to refer to music in terms of keywords such as "sad", "fast" or "dynamic", rather than to define music parameters to a specific scale and meter, which is the case for Aiva.

\section{DISCUSSION}

In this study we have adopted a user centered approach to the design of a music generation system based on "creation by example". Through several user studies we observed that there is a need for simpler and more easily accessible tools for generating music for video. We developed a system that allows for curation of AI music based on a UI paradigm allowing for interactive and iterative mixing of AI-generated material. Our results prove that our designed system succeeded in meeting user needs as specified in user studies. Moreover, our system was perceived as easy and enjoyable to use, require little learning time, and produce satisfying musical outcomes by the majority of the participants. The proposed system can be useful to video creators since it enables music creation for video without requiring tedious search and editing processes.

User studies shed light on the fact that it is important to balance the control assigned to the AI versus the human input; there is a distinction between tasks that users want to leave to the AI, versus tasks that they want to perform themselves. Our work raises interesting questions about autonomy, agency and authorship in human-AI interaction in creative practice. In our case, users did not want the system to have too much control over the process of creating music. This since they did not feel that they had actually created any music material themselves if too much control was assigned to the AI. This is an interesting finding, considering that recent advancements in deep learning for music creation largely has focused on autonomous generation.

The system proposed in this paper reduces the need for specific domain knowledge in machine learning or algorithmic methods for music generation; it allows users to translate a musical idea to sounds without requiring them to have any formal musical training, thereby making music creation easily accessible through design. In this way, design by example is a novel way of providing input to a music generation process. We believe that this design metaphor could be employed also in other areas, for example in generative design for games, visual art, sketching apps and other creative tools. Generative design methods often produce a lot of AI-generated material that needs to be curated by a human agent. There is a need for new interfaces for discovering the range of material produced by such AI systems. The mix-and-match paradigm proposed in this paper can be one way of exploring a vast dimension space of AI-generated material.

We want to acknowledge that there are limitations of AI music when it comes to the aesthetic quality of the musical outcome. For our particular case, the main limitation in this regard was for the generated drum patterns of IBM Watson Beat, and we therefore opted for predefined drum loop sample libraries. Mixed-initiative interactions such as the ones described in this paper should be designed to promote successful collaboration between a human and an AI system. This way, the human agent can curate the material generated by the system's algorithmic counterparts. While our current system design relies on MIDI, our design is modular in the sense that machine learning algorithms could be updated and replaced while the rest of the system remains intact. For this purpose, other methods (see e.g. $[58,71]$ ) resulting in more aesthetically appealing results could serve as components for the development of a future prototype. Another suggestion for future development is to implement an interactive multitrack timeline with temporal synchronization features based on extracted video features.

\section{CONCLUSIONS}

This paper describes a music creation paradigm informed by a user centered design approach. We present a prototype system that allows users to create songs in collaboration with an AI music system by providing an example reference of a wellknown song and then interactively mix AI-generated material. Findings and insights from user studies involving more than 104 video creators support the effectiveness of our approach. The software addresses issues of finding music for videos by allowing users to create their own unique sounds based on a reference song. This work is unique in the sense that it is the first time that design by example is applied in the context of music generation. Our research raises interesting questions about the level of control assigned to users versus AI in mixedinitiative interactions, findings that could be applied in other areas of research related to human-AI interaction. With few existing evaluation frameworks for human-AI interaction and an increasing presence of $\mathrm{AI}$ in $\mathrm{HCI}$, we believe that research on novel interfaces for mixed-initiative interactions should be encouraged and hope to promote such discussions with this work. 


\section{REFERENCES}

[1] Adobe. 2019. Adobe Audition. (2019). https://www. adobe. com/se/products/audition.html.

[2] Kat Agres, Jamie Forth, and Geraint A Wiggins. 2016. Evaluation of Musical Creativity and Musical Metacreation Systems. Computers in Entertainment (CIE) 14, 3 (2016), 3.

[3] Google Research / AI. 2019. Magenta. (2019). https://ai . google/research/teams/brain/magenta/.

[4] JE Allen, Curry I Guinn, and E Horvtz. 1999. Mixed-Initiative Interaction. IEEE Intelligent Systems and their Applications 14, 5 (1999), 14-23.

[5] Giuseppe Amato, Malte Behrmann, Frédéric Bimbot, Baptiste Caramiaux, Fabrizio Falchi, Ander Garcia, Joost Geurts, Jaume Gibert, Guillaume Gravier, Hadmut Holken, and others. 2019. AI in the Media and Creative Industries. (2019).

[6] Vanessa Theme Ament. 2014. The Foley Grail: The Art of Performing Sound for Film, Games, and Animation. Routledge, Burlingto, MA, USA.

[7] Saleema Amershi, Dan Weld, Mihaela Vorvoreanu, Adam Fourney, Besmira Nushi, Penny Collisson, Jina Suh, Shamsi Iqbal, Paul N Bennett, Kori Inkpen, and others. 2019. Guidelines for Human-AI Interaction. In Proceedings of the 2019 CHI Conference on Human Factors in Computing Systems. ACM, ACM, New York, NY, USA, 3.

[8] Christopher Ariza. 2009. The Interrogator as Critic: The Turing Test and the Evaluation of Generative Music Systems. Computer Music Journal 33, 2 (2009), 48-70.

[9] Gérard Assayag, Camilo Rueda, Mikael Laurson, Carlos Agon, and Olivier Delerue. 1999. Computer-Assisted Composition at IRCAM: From PatchWork to OpenMusic. Computer Music Journal 23, 3 (1999), 59-72.

[10] Francisco Bernardo, Michael Zbyszynski, Rebecca Fiebrink, and Mick Grierson. 2017. Interactive Machine Learning for End-User Innovation. In 2017 AAAI Spring Symposium Series. The AAAI Press, Palo Alto, CA, USA, 369-375.

[11] J Bharucha. 1988. Neural Net Modeling of Music. In Proceedings of the First Workshop on Artificial Intelligence and Music. American Association for Artifical Intelligence, Menlo Park, CA, USA, 173-182.

[12] Margaret A. Boden. 1998a. Creativity and Artificial Intelligence. Artificial Intelligence 103, 1 (1998), 347 356. http://www. sciencedirect. com/science/article/ pii/S0004370298000551 Artificial Intelligence 40 Years Later.

[13] Margaret A Boden. 1998b. Creativity and Artificial Intelligence. Artificial Intelligence 103, 1-2 (1998), 347-356.
[14] Jean-Pierre Briot, Gaëtan Hadjeres, and François Pachet. 2017. Deep Learning Techniques for Music Generation A Survey. (2017).

[15] Kris Bryden. 2007. Developing Metrics for Evolving a Musically Satisfying Result from a Population of Computer Generated Music Compositions. In 2007 IEEE Symposium on Computational Intelligence in Image and Signal Processing. IEEE, Honolulu, HI, USA, 171-176.

[16] Cognitive Catalyst. 2019. Watson Beat. (2019). https://github.com/cognitive-catalyst/watson-beat.

[17] Elizabeth Charters. 2003. The Use of Think-Aloud Methods in Qualitative Research - An Introduction to Think-Aloud Methods. Brock Education: A Journal of Educational Research and Practice 12, 2 (2003), 68-82.

[18] Simon Colton, Ramon López de Mántaras, and Oliviero Stock. 2009. Computational Creativity: Coming of Age. AI Magazine 30, 3 (2009), 11-11.

[19] David Cope. 1989. Experiments in Musical Intelligence (EMI): Non-Linear Linguistic-Based Composition. Journal of New Music Research 18, 1-2 (1989), 117-139.

[20] David Cope. 2000. The Algorithmic Composer. Vol. 16. AR Editions, Inc., Madison, Wisconsin, USA.

[21] Boomy Corporation. 2019. Make Instant Music with Artificial Intelligence. (2019). https://boomy.com/.

[22] Cycling'74. 2019. Cycling'74 - Tools For Sound, Graphics and Interactivity. (2019). https://cycling74.com/.

[23] Ramon Lopez De Mantaras and Josep Lluis Arcos. 2002. AI and Music: From Composition to Expressive Performance. AI magazine 23, 3 (2002), 43-43.

[24] Steven P Dow, Alana Glassco, Jonathan Kass, Melissa Schwarz, Daniel L Schwartz, and Scott R Klemmer. 2010. Parallel Prototyping Leads to Better Design Results, More Divergence, and Increased Self-Efficacy. ACM Transactions on Computer-Human Interaction (TOCHI) 17, 4 (2010), 18.

[25] ElectronJS. 2019. ElectronJS - Build Cross Platform Desktop Apps with JavaScript, HTML, and CSS. (2019). https://electronjs.org//.

[26] Jose D Fernández and Francisco Vico. 2013. AI Methods in Algorithmic Composition: A Comprehensive Survey. Journal of Artificial Intelligence Research 48 (2013), 513-582.

[27] Rebecca Fiebrink, Baptiste Caramiaux, R Dean, and A McLean. 2018. The Machine Learning Algorithm as Creative Musical Tool. Oxford University Press, New York, NY, USA, Chapter 12, 181-208.

[28] Filmstro. 2019. Filmstro - Music That Moves. (2019). https://filmstro.com/. 
[29] Dorien Herremans and Elaine Chew. 2017. MorpheuS: Generating Structured Music with Constrained Patterns and Tension. IEEE Transactions on Affective Computing (2017), 510-523.

[30] Hexachords. 2019. Orb Composer. (2019). https://www. orb-composer. com/.

[31] IBM. 2019. IBM Watson Beat. (2019). https://www.ibm.com/case-studies/ibm-watson-beat.

[32] Muzeek Inc. 2019. Muzeek Pro. (2019). https://www . getmuzeek. com/home.

[33] Bruce L. Jacob. 1996. Algorithmic Composition as a Model of Creativity. Organised Sound 1, 3 (1996), 157-165.

[34] Lilong Jiang, Protiva Rahman, and Arnab Nandi. 2018. Evaluating Interactive Data Systems: Workloads, Metrics, and Guidelines. In Proceedings of the 2018 International Conference on Management of Data. ACM, ACM, New York, NY, USA, 1637-1644.

[35] Simon C Jones and Thomas G Schumacher. 1992. Muzak: On Functional Music and Power. Critical Studies in Media Communication 9, 2 (1992), 156-169.

[36] Jukedeck. 2019. Jukedeck. (2019). https://www.jukedeck.com/.

[37] SmartSound LCC. 2019. Royalty Free music and Incredible ways to customize it. (2019). https://www. smartsound. com/.

[38] Yuli Levtov. 2018. Algorithmic Music for Mass Consumption and Universal Production. In The Oxford Handbook of Algorithmic Music. Oxford University Press, New York, NY, USA.

[39] Jen-Chun Lin, Wen-Li Wei, and Hsin-Min Wang. 2015. EMV-Matchmaker: Emotional Temporal course Modeling and Matching for Automatic Music Video Generation. In Proceedings of the 23rd ACM International conference on Multimedia. ACM, ACM, New York, NY, USA, 899-902.

[40] Jen-Chun Lin, Wen-Li Wei, and Hsin-Min Wang. 2016. DEMV-matchmaker: Emotional Temporal Course Representation and Deep Similarity Matching for Automatic Music Video Generation. In Proceedings of ICASSP. IEEE, Shanghai, China, 2772-2776.

[41] Jen-Chun Lin, Wen-Li Wei, James Yang, Hsin-Min Wang, and Hong-Yuan Mark Liao. 2017. Automatic Music Video Generation Based on Simultaneous Soundtrack Recommendation and Video Editing. In Proceedings of the 25th ACM international conference on Multimedia. ACM, ACM, New York, NY, USA, 519-527.

[42] Levelo Music Group LLC. 2019a. Soundstripe Unlimited Music for Video. (2019).

https://soundstripe.com/.
[43] Marmoset LLC. 2019b. Marmoset Music. (2019). https://www . marmosetmusic. com/.

[44] SmartSound LLC. 2019c. Sonicfire Pro 6 - The Fastest Way to Customize Music to Your Videos. (2019). https://www. smartsound.com/sonicfire/.

[45] Envato Pty Ltd. 2019. AudioJungle. (2019). https://audiojungle.net/.

[46] L Manovich. 2018. AI Aesthetics. Strelka Press, Moscow, Russia.

[47] John Maurer. 1999. A Brief History of Algorithmic composition. (1999).

[48] Jon McCormack and Mark d'Inverno. 2014. On the Future of Computers and Creativity. In AISB 2014 Symposium on Computational Creativity. Society for the Study of Artificial Intelligence and the Simulation of Behaviour, London, UK, 9.

[49] Alex McLean and Roger T Dean. 2018a. Musical Algorithms as Tools, Languages, and Partners: A Perspective. Oxford University Press, New Yor, NY, USA, 3-15.

[50] Alex McLean and Roger T Dean. 2018b. The Oxford Handbook of Algorithmic Music. Oxford University Press, New York, NY, USA.

[51] Eduardo Reck Miranda. 2013. Readings in Music and Artificial Intelligence. Routledge, New York and London.

[52] Amper Music. 2019a. (2019). https://www . ampermusic. com/.

[53] Amper Music. 2019b. Amper A.I. vs. Stock Results. (2019). https://www . ampermusic. com/docs/veritonic_ study--amper_v_stock.pdf.

[54] PG Music. 2019c. Band-in-a-Box. (2019). https://www.pgmusic.com/.

[55] MusicBed. 2019. MusicBed. (2019). https://www.musicbed.com/.

[56] Gerhard Nierhaus. 2009. Algorithmic Composition: Paradigms of Automated Music Generation. Springer Science \& Business Media, New York, NY, USA.

[57] NIME. 2019. The International Conference on New Interfaces for Musical Expression. (2019).

https://www.nime.org/.

[58] Aaron van den Oord, Sander Dieleman, Heiga Zen, Karen Simonyan, Oriol Vinyals, Alex Graves, Nal Kalchbrenner, Andrew Senior, and Koray Kavukcuoglu. 2016. Wavenet: A Generative Model for Raw Audio. (2016).

[59] Alexandre Papadopoulos, Pierre Roy, and François Pachet. 2016. Assisted Lead Sheet Composition Using FlowComposer. In Proceedings of International Conference on Principles and Practice of Constraint Programming. Springer, Toulouse, France, 769-785. 
[60] George Papadopoulos and Geraint Wiggins. 1999. AI Methods for Algorithmic Composition: A Survey, a Critical View and Future Prospects. In AISB Symposium on Musical Creativity, Vol. 124. Edinburgh, UK, 110-117.

[61] Richard Scheines. 1988. Automating Creativity. In Aspects of Artificial Intelligence. Springer, 339-365.

[62] Adriana Schulz, Ariel Shamir, David IW Levin, Pitchaya Sitthi-Amorn, and Wojciech Matusik. 2014. Design and Fabrication by Example. ACM Transactions on Graphics (TOG) 33, 4 (2014), 62.

[63] Rajiv Ratn Shah, Yi Yu, and Roger Zimmermann. 2014. Advisor: Personalized Video Soundtrack Recommendation by Late Fusion with Heuristic Rankings. In Proceedings of the 22nd ACM international conference on Multimedia. ACM, ACM, New York, NY, USA, 607-616.

[64] Kristina Shea, Robert Aish, and Marina Gourtovaia. 2005. Towards Integrated Performance-Driven Generative Design Tools. Automation in Construction 14, 2 (2005), 253-264.

[65] Shutterstock. 2019. Premium Beat - Score the Perfect Music. (2019). https://www.premiumbeat.com/.

[66] Epidemic Sound. 2019. Epidemic Sound. (2019). https://www. epidemicsound.com/.

[67] Thomas Strothotte and Stefan Schlechtweg. 2002. Non-Photorealistic Computer Graphics: Modeling, Rendering, and Animation. Morgan Kaufmann, San Francisco, CA, USA.
[68] Aiva Technologies. 2019. The Artificial Intelligence Composing Emotional Soundtrack Music. (2019). https://www. aiva.ai/.

[69] Benjamin Tissot. 2019. Bensound's Royalty Free Music. (2019). https://www. bensound.com/.

[70] UserTesting. 2019. UserTesting. (2019). https://www . usertesting.com/.

[71] Sean Vasquez and Mike Lewis. 2019. MelNet: A Generative Model for Audio in the Frequency Domain. (2019).

[72] Ju-Chiang Wang, Yi-Hsuan Yang, I-Hong Jhuo, Yen-Yu Lin, Hsin-Min Wang, and others. 2012. The Acousticvisual Emotion Guassians Model for Automatic Generation of Music Video. In Proceedings of the 20th ACM international conference on Multimedia. ACM, ACM, New York, NY, USA, 1379-1380.

[73] Robert Philip Weber. 1990. Basic Content Analysis. Sage, Newbury Park, California.

[74] Ariel Weingarten, Ben Lafreniere, George Fitzmaurice, and Tovi Grossman. 2019. DreamRooms: Prototyping Rooms in Collaboration with a Generative Process. In Proceedings of Graphics Interface 2019 (GI 2019). Canadian Information Processing Society, Mississauga, Ontario, Canada, 9.

[75] Iannis Xenakis. 1992. Formalized Music: Thought and Mathematics in Composition. Pendragon Press, Stuvesant, NY, USA.

[76] Youtube. 2019. Youtube Audio Library. (2019). https://www. youtube. com/audiolibrary/. 